\title{
MRI Evaluation of Global Developmental Delay: A Retrospective Study
}

\author{
Hafiz Habibullah Raidah Albaradie Shahid Bashir \\ Pediatric Neurology, Neuroscience Center, King Fahad Specialist Hospital Dammam, Dammam, Saudi Arabia
}

\section{Keywords}

Developmental · Disability · Medicine · Neurology ·

Radiology

\begin{abstract}
Introduction: Global developmental delay (GDD) is defined as a significant delay in one or more developmental domains. Aims and Objectives: To study the prevalence of normal and abnormal magnetic resonance imaging (MRI) in pediatric patients presenting with GDD, and how abnormal MRI helps in the diagnosis of children with GDD. Materials and Methods: This was a retrospective observational study conducted at the King Fahad Specialist Hospital Dammam. MRI of the brain was conducted on 170 patients who were referred by the Pediatric Neurology Department between February 2016 and April 2018. Results: Normal MRI findings were seen in $45.3 \%$ and $54.7 \%$ had abnormal findings. The ventricles and white matter, mainly the corpus callosum, were the most commonly affected anatomical structures. In 15 (16\%) patients, MRI enabled a direct diagnosis, and in 22 (23.6\%) MRI suggested a diagnosis which was confirmed by further investigation. Conclusion: The clinical diagnosis of GDD should not be the end point, but rather a springboard for an effective search for causal factors. MRI is the best investigation with a high yield in such patients.
\end{abstract}

(c) 2020 The Author(s)

Published by S. Karger AG, Basel

\section{KARGER}

karger@karger.com www.karger.com/dmj

\section{Introduction}

Global developmental delay (GDD) is a continuous process which begins from conception and continues up till maturity. During this process, genetic, environmental, and nutritional factors as well as chronic diseases can have adverse effects on developmental milestones in 4 domains: gross motor, fine motor, social, and language skills [1]. The degree of developmental delay is further subclassified as mild (functional age $<33 \%$ below chronological age), moderate (functional age $34-66 \%$ of chronological age) and severe (functional age $>66 \%$ of chronological age) [2]. GDD is not considered as a disease or diagnosis but rather as a symptom or clinical presentation [1-6].

Though no accurate records are available, it is believed that patients with GDD constitute nearly $5-10 \%$ of those presenting as outpatients at various medical centers [7]. The developmental delay may become evident during infancy or early childhood, but is more apparent and therefore more often diagnosed in the early school years [8]. Establishing a diagnosis enables clinicians to define treatment options and conduct surveillance for known complications as well as provide prognosis and conditionspecific family support (including family-planning choices). This ensures the best overall outcomes for the child and their family [9]. A diagnosis can provide an explanation and a source of closure or acceptance for parents; it

Hafiz Habibullah

Neuroscience Center

King Fahad Specialist Hospital Dammam

Dammam 31444 (Saudi Arabia)

hafiz.habibullah@kfsh.med.sa 
Table 1. Age and sex distribution of study population with normal and abnormal MRI

\begin{tabular}{lccccc}
\hline Parameter & \multicolumn{2}{c}{ Abnormal $(n=93)$} & & \multicolumn{2}{c}{ Normal $(n=77)$} \\
& $n$ & $\%$ & & $n$ & $\%$ \\
\hline Age & & & & \\
$\quad$ 3 months - 1 year & 7 & 7.5 & & 10.3 \\
1-2 years & 14 & 15.0 & & 11.6 \\
2-5 years & 27 & 29.0 & & 23 & 29.8 \\
5-8 years & 21 & 22.5 & & 24 & 31.1 \\
8-12 years & 24 & 25.8 & & 13 & 16.8 \\
Sex & & & & \\
$\quad$ Male $(n=93)$ & 54 & 58.0 & & 39 & 42.0 \\
$\quad$ Female $(n=77)$ & 39 & 50.6 & 38 & 49.4 \\
\hline
\end{tabular}

also stops clinicians advancing to potentially more expensive and invasive tests [10-12].

Brain MRI is one of the major investigations conducted on these patients and, based on previous studies, about $60 \%$ of cases have abnormal brain MRI $[13,14]$. Clinical findings can sometimes lead to diagnosis, but in most cases MRI is necessary to get an actual picture of the abnormality. This facilitates accurate diagnosis which further helps the clinician in properly treating the patient.

This study aimed to characterize the structural anomalies visible on MRI of the brain and the prevalence of normal and abnormal MRI in pediatric patients presenting with GDD.

\section{Materials and Methods}

We conducted a detailed retrospective chart review of 170 children diagnosed with GDD in the Pediatric Neurology and Development Assessment Clinic at the King Fahad Specialist Hospital Dammam (KFSHD) between Feb 2016 and April 2018. Referrals to the clinic derive mainly from pediatricians, family physicians, and pediatric neurology in the eastern province region of Saudi Arabia.

All children referred to the clinic undergo a formal multidisciplinary developmental assessment (a complete history and a developmental examination). Both sexes were included in the study. The sequences used were: axial T1TSE, T2TSE, T2 FLAIR, EP2D diffusion, T2TIRM, and PDTSE; coronal T1TIR and T2TSE; and sagittal T1TSE.

\section{Inclusion and Exclusion Criteria}

Children aged 3 months to 12 years presenting with developmental delay were enrolled in the study. Patients with autism and who were non-cooperative in the developmental assessment were excluded. When the dataset of the patient (as designed in the clinical evaluation sheet) was not complete, they were also excluded.

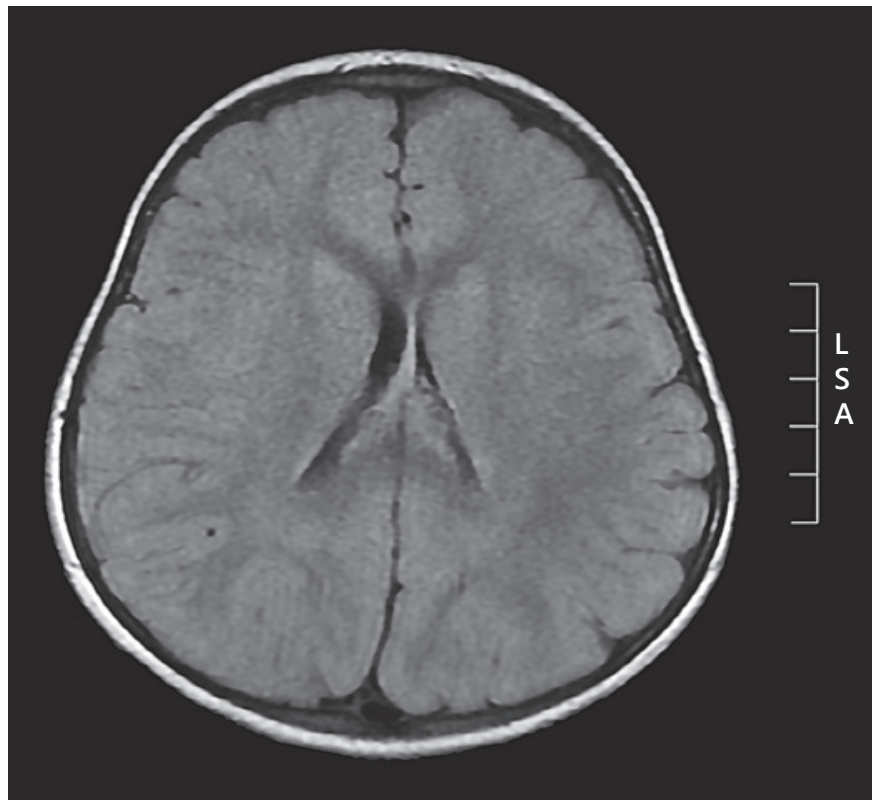

Fig. 1. T2-weighted axial image showing a normal brain.

\section{Neuroimaging}

After conducting MRI of the brain, the images were examined and the following structures were systematically evaluated according to the protocol of Widjaja et al. [9].

- Ventricles: size and morphology.

- Corpus callosum: thickness and morphology.

- Gray and white matter: the sulcation and gyration of the gray matter based on normal MRI brain anatomy.

- Basal ganglia: morphology.

- Brain stem: morphology.

- Cerebellum: morphology.

- A structure was considered dysplastic if disorganized in development, e.g., an abnormal folial pattern was evident or heterotopic nodules of gray matter were present.

\section{Statistical Analysis}

All data gathered were analyzed using SPSS software v22.0 (SPSS Inc., Chicago). $p \leq 0.05$ was considered statistically significant.

\section{Results}

Normal MRI brain findings were seen in 77 (45.3\%) pediatric patients presenting with GDD (Table 1; Fig. 1). These children were advised to undergo further evaluation to determine the etiology. An abnormal morphological appearance was detected in the remaining 93 cases (54.7\%) (Table 1; Fig. 2). Most of the children with abnormal MRI findings were in the age group of $2-5$ years (29\%), with the 
Fig. 2. T1-weighted mid-saggital image showing an open-lip schizencephaly with agenesis of the corpus callosum.

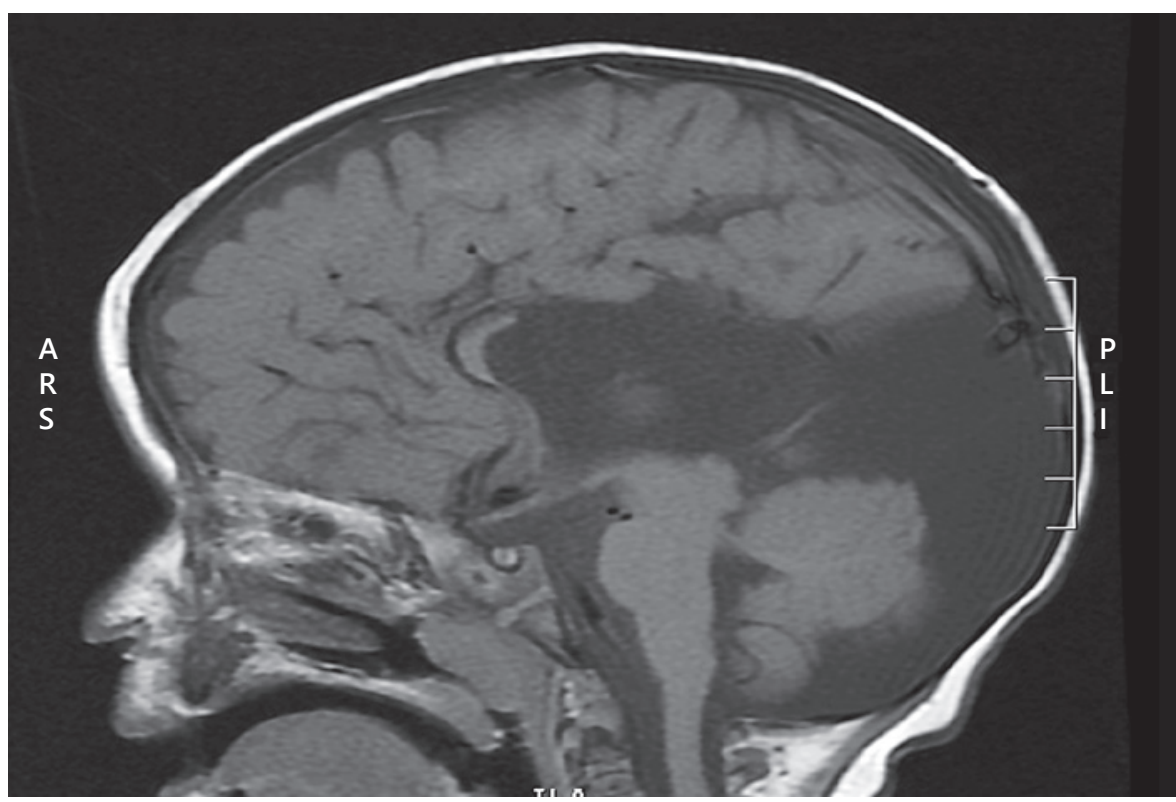

next peak at $8-12$ years $(25.8 \%)$. Males $(58.0 \%)$ were slightly more in number than females $(50.6 \%)$. In 15 patients (16\%), MRI enabled a direct diagnosis, and in 22 (23.6.8\%) MRI suggested the diagnosis which was then confirmed by further investigation. The remaining 56 patients (60.2\%) had no specific abnormalities on MRI (Fig. 3).

\section{Discussion}

There is a lack of studies in Saud Arabia about the prevalence of GDD, but it seems to be in the range of $1.5-2.5 \%$ in children under 2 years of age $[15,16]$. We made an evaluation of GDD in 170 consecutive pediatric patients aged 3 months to 12 years. The proportion of children with abnormal MRI findings was $54.7 \%$. Similar MRI results have been reported by Momen et al. [1], Shevell et al. [3], Pandey et al. [6], Koul et al. [7], Battaglia et al. [8], and Widjaja et al. [9], i.e., 58.6, 65.5, 63.8, 71.8, 80.8 , and $84 \%$, respectively. This wide range could be due to differences in patient selection criteria and awareness about investigating such children across different population groups. Neuroimaging by means of MRI has an indispensable role in evaluating a child with GDD, and the etiological yield can be increased if other associated clinical and neurological signs and symptoms are taken into the inclusion criteria $[3,9]$. The age of presentation in our patients differed from Momen et al. [1] but sex incidence was similar.

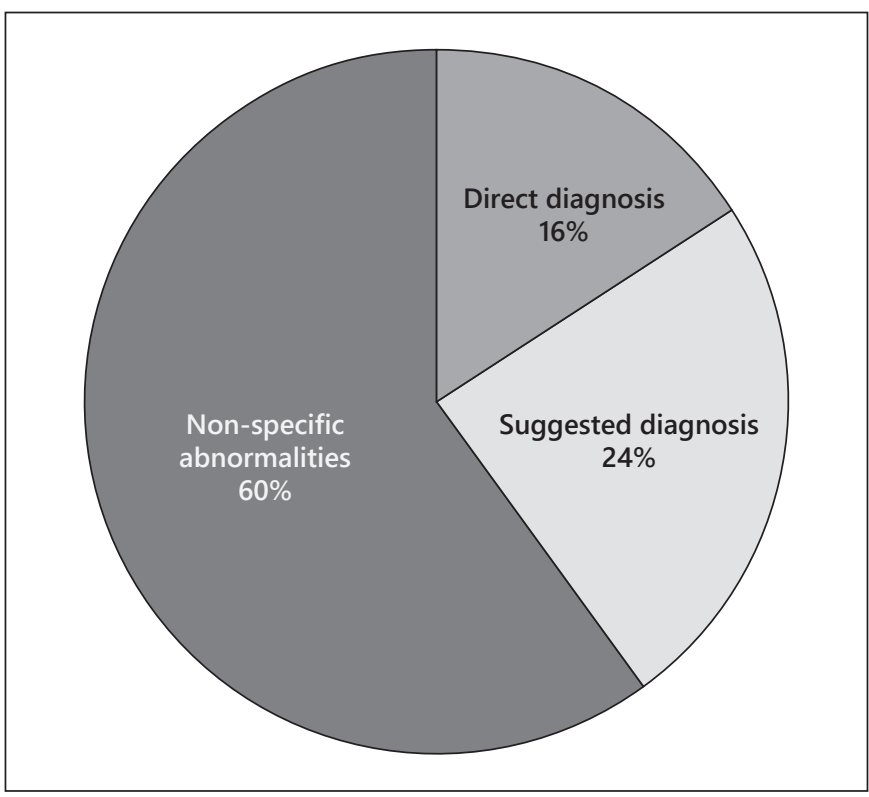

Fig. 3. Brain MRI enabled a direct diagnosis in $16 \%$ of patients and suggested the etiology in $24 \%$. This guided the treating clinician in planning further investigations to establish diagnosis and proper management.

The 93 cases with abnormal MRI were evaluated for involvement of various anatomical structures, with the ventricles and white matter, mainly the corpus callosum, being the most common. Widjaja et al. [9] studied 90 such 
children and found that the ventricles and corpus callosum were the most commonly involved; the involvement of other structures was similar to in our study.

MRI is an important part of the comprehensive evaluation of children with GDD, as many contributing factors, namely specific etiologic and pathophysiologic conditions, can be detected easily [12-18]. Evidence supports that early diagnosis and treatment of developmental disorders leads to improvement.

\section{Conclusion}

MRI evaluation of the brain contributes to the diagnosis of GDD. GDD has a wide spectrum of etiologies ranging from normal to abnormal. MRI study of the brain helps the clinician to make a proper diagnosis. This facilitates the appropriate treatment of the child, and also the counseling of the parents with regard to outcomes and the risk of recurrence in siblings and subsequent generations. The chance of improving diagnostics increases with brain MRI. Advances in MRI technology like functional MRI, MR spectroscopy, diffusion tensor imaging, and tractography especially in the structurally normal brain of such children, would provide even more information.

\section{Acknowledgement}

The authors would like to thank Mary J. Chemmandakaran, the epilepsy coordinator, for her administrative help.

\section{Statement of Ethics}

The investigation was carried out in accordance with the latest version of the Declaration of Helsinki and was approved by the local review board, the KFSHD ethics committee. All participants or their parents gave their written informed consent prior to enrollment in the study.

\section{Disclosure Statement}

None of the authors have potential conflicts of interest to be disclosed.

\section{Funding Sources}

No funds were available or need to be reported for this work.

\section{Author Contributions}

H.H. and R.A. designed the study; S.B. and H.H. collected the data; S.B. and R.A. analyzed the data; and all 3 authors wrote and reviewed the manuscript.

\section{References}

1 Momen AA, Jelodar G, Dehdashti H. Brain Magnetic Resonance Imaging Findings in Developmentally Delayed Children. Int J Pediatr. 2011;2011:386984.

2 Battaglia A, Carey JC. Diagnostic evaluation of developmental delay/mental retardation: an overview. Am J Med Genet C Semin Med Genet. 2003 Feb;117C(1):3-14.

3 Shevell M, Ashwal S, Donley D, Flint J, Gingold M, Hirtz D, et al.; Quality Standards Subcommittee of the American Academy of Neurology; Practice Committee of the Child Neurology Society. Practice parameter: evaluation of the child with global developmental delay: report of the quality standards subcommittee of the American Academy of Neurology and The Practice Committee of the Child Neurology Society. Neurology. 2003 Feb;60(3):36780.

4 McDonald LA, Rennie AC. Investigating developmental delay / impairment. Paediatr Child Health. 2011;21(10):443-7.

5 Petersen MC, Kube DA, Palmer FB. Classification of developmental delays. Semin Pediatr Neurol. 1998 Mar;5(1):2-14.
6 Pandey A, Phadke SR, Gupta N, Phadke RV. Neuroimaging in mental retardation. Indian J Pediatr. 2004 Mar;71(3):203-9.

7 Koul R, Al-Yahmedy M, Al-Futaisi A. Evaluation children with global developmental delay: a prospective study at sultan qaboos university hospital, oman. Oman Med J. 2012 Jul;27(4):310-3.

8 Battaglia A, Bianchini E, Carey JC. Diagnostic yield of the comprehensive assessment of developmental delay/mental retardation in an institute of child neuropsychiatry. Am J Med Genet. 1999 Jan;82(1):60-6.

9 Widjaja E, Nilsson D, Blaser S, Raybaud C. White matter abnormalities in children with idiopathic developmental delay. Acta Radiol. 2008 Jun;49(5):589-95.

10 Patel S, Barkovich AJ. Analysis and classification of cerebellar malformations. AJNR Am J Neuroradiol. 2002 Aug;23(7):1074-87.

11 Williams HJ. Imaging the child with developmental delay. Imaging. 2004;16(2):174-85.

12 Rivkin MJ. Developmental neuroimaging of children using magnetic resonance techniques. Ment Retard Dev Disabil Res Rev. 2000;6(1):68-80.
13 Moes P, Schilmoeller K, Schilmoeller G. Physical, motor, sensory and developmental features associated with agenesis of the corpus callosum. Child Care Health Dev. 2009 Sep; 35(5):656-72.

14 McDonald L, Rennie A, Tolmie J, Galloway P, McWilliam R. Investigation of global developmental delay. Arch Dis Child. 2006 Aug; 91(8):701-5.

15 Schaefer GB, Bodensteiner JB. Radiological findings in developmental delay. Semin Pediatr Neurol. 1998 Mar;5(1):33-8.

16 Walters AV. Developmental Delay Causes and Investigations. Adv Clin Neurosci Rehabil. 2010;10(2):32-4.

17 Harbord MG, Finn JP, Hall-Craggs MA, Robb SA, Kendall BE, Boyd SG. Myelination patterns on magnetic resonance of children with developmental delay. Dev Med Child Neurol. 1990 Apr;32(4):295-303.

18 Curry CJ, Stevenson RE, Aughton D, Byrne J, Carey JC, Cassidy S, et al. Evaluation of mental retardation: recommendations of a consensus conference. Am J Med Genet. 1997 Nov;72(4):468-77. 